\title{
Sistema Automatizado de Controle de Abastecimento de Água Proveniente de Poços Artesianos com Monitoramento Remoto
}

\author{
Automated water supply control system from artesian wells with remote monitor
}

Adailton Medeiros Rodrigues de Oliveira ${ }^{1}$ (i) orcid.org/0000-0002-5386-9979

\author{
Gustavo Oliveira Cavalcanti ${ }^{1}$ (D) orcid.org/0000-0001-9859-908X \\ ${ }^{1}$ Escola Politécnica de Pernambuco, Universidade de Pernambuco, Recife, Brasil \\ E-mail do autor principal: Adailton Medeiros Rodrigues de Oliveira adailton.medeiros@gmail.com
}

\begin{abstract}
Resumo
Este trabalho descreve a construção de hardware e software de baixo custo para controlar e monitorar, através da Internet e/ou uma rede local, o abastecimento de reservatórios residenciais com água proveniente de poços artesianos. O objetivo do sistema é evitar o desperdício da água pelo transbordamento dos reservatórios, bem como reduzir o consumo de energia com a bomba do motor elétrico. O sistema é composto por sensores eletromecânicos de nível, rotâmetro para medição de vazão, válvulas solenoides para direcionamento de fluxo e um módulo de desenvolvimento. $O$ sistema eletrônico desenvolvido emprega o chip ESP8266EX, que possui diversos recursos que facilitam a filosofia da Internet das Coisas (IOT). O software de controle foi desenvolvido em linguagem Lua e o aplicativo de monitoramento através de smartphones foi criado com a ferramenta de desenvolvimento App Inventor. O software em conjunto com o aplicativo permitem o monitoramento e controle dos níveis dos reservatórios de forma simples e econômica.
\end{abstract}

Palavras-Chave: Poços artesiano, monitoramento remoto, ESP8266, IoT, Lua, App Inventor.

\begin{abstract}
This paper describes the construction of low cost hardware and software to control and monitor the supply of residential reservoirs with water from artesian wells through the Internet and / or local area network. The goal is to avoid wasting water by trans-embroidery reservoirs, as well as reduce energy consumption with the electric motor pump. The system consists of electromechanical level sensors, flowmeter for flow measurement, solenoid valves for flow direction and a development module. The electronic system developed employs the ESP8266EX chip, which has several features that facilitate the Internet philosophy of Things (IoT). The control software was developed in Lua language and the smartphone monitoring application was created with the App Inventor development tool. The software in conjunction with the application allows the monitoring and control of reservoir levels simply and economically.
\end{abstract}


Key-words: Corrosion; Diffusion of Chloride Ions; Durability; Additions in Cement.

\section{Introdução}

A deficiência no sistema de abastecimento de água da RMR (Região Metropolitana do Recife) fez com que houvesse um crescimento desordenado na perfuração e construção de poços artesianos e consequentemente um impacto na qualidade das nossas águas subterrâneas, além da diminuição do volume dos lençóis freáticos [1].

A retirada de água dos poços artesiano ocorre, normalmente, através de uma moto bomba de sucção que pode ser operada manualmente ou através de chaves de nível, fazendo com que o sistema ligue quando há nível baixo e desligue quando o reservatório está cheio, desconsiderando possíveis falhas do sistema como por exemplo, entrada de ar nas tubulação de sucção do poço, ocasionando aquecimento na bomba. Na operação manual o equipamento é ligado quando há a necessidade de abastecer devido a falta d'água e é desligado quando há o transbordamento do reservatório d'água como ilustrado na Figura 1, ocasionando o desperdício de água e energia elétrica.

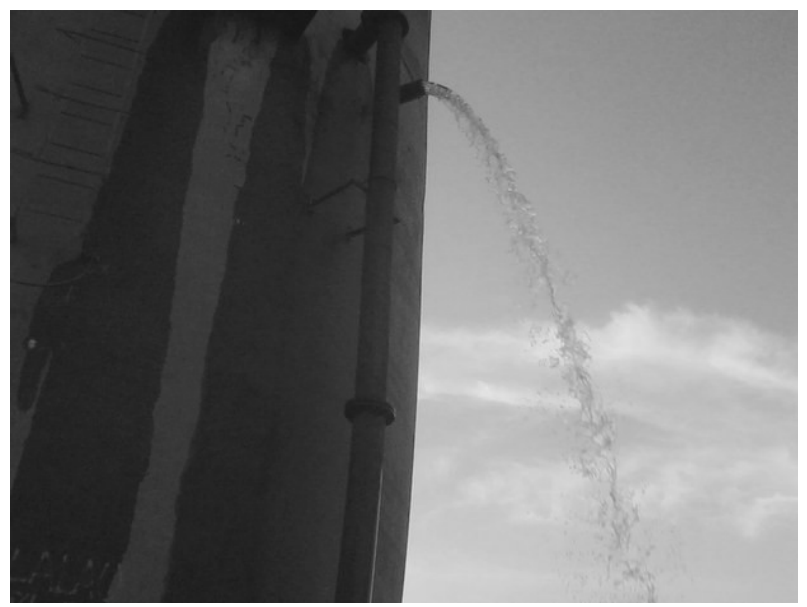

Figura 1: Reservatório extravasando.

A automação residencial passou a ser uma realidade mais acessível a todas as classes sociais devido a diversidade e a diminuição dos valores dessa tecnologia. A Internet está presente na maioria das residências e os aparelhos telefônicos do tipo 129
SmartPhone são, hoje, uma ferramenta essencial no dia a dia para a comunicação, lazer, aprendizagem, informação e se tornam também uma chave mestra para o controle de dispositivos eletrônicos como TV's, HomeTheather, Condicionadores de AR entre diversos outros eletrodoméstico que conseguem se comunicar com a Internet.

O uso destas tecnologias está se tornando cada vez mais popular e de baixo custo [2][3], os aplicativos para dispositivos móveis são capazes de fornecer informações em tempo real dos sensores quando conectados à Internet [4][5].

Este trabalho reúne a facilidade da conectividade dos aparelhos eletrônicos com a necessidade de se evitar o desperdício de água. Sendo assim, foi desenvolvida uma aplicação para controlar um sistema de abastecimento através de um motor que bombeia a água de um poço artesiano, envia para quatro reservatórios quando os níveis chegam ao seu valor mínimo e interrompe o abastecimento quando estão completamente cheios. O sistema evita 0 transbordamento e consequentemente o desperdício de água e energia. A seguir descreve-se o cenário de funcionamento do sistema e sua aplicação.

\subsection{O Cenário e seu Funcionamento}

O projeto foi dimensionado para atender o cenário ilustrado na Figura 2. A água é retirada do poço por sucção e direcionada aos tanques TQ1,TQ2,TQ3 e a cisterna, identificada como TQ4, por meio das válvulas VALV 01,VALV 02,VALV 03 e VALV 04, respectivamente, conforme ilustrado na Figura 2. 
FLUXOGRAMA DO PROCESSO

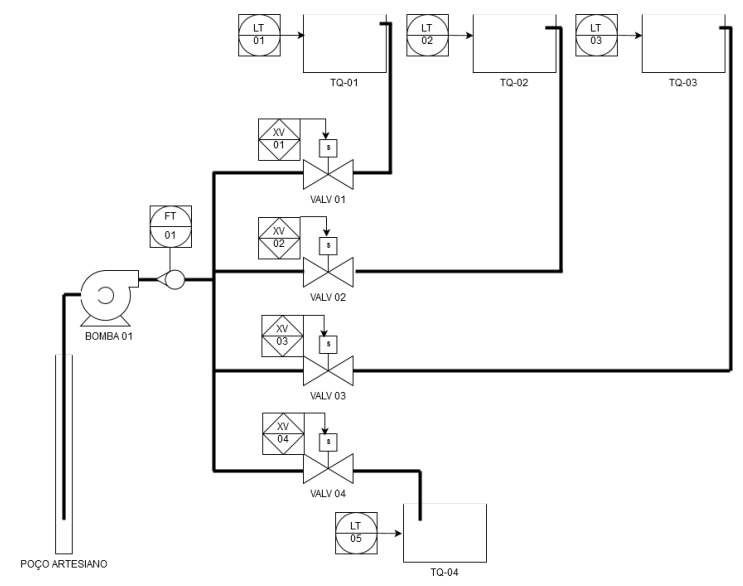

Figura 2: Cenário a ser controlado pela aplicação.

O sistema possui dois modos de funcionamento: manual e automático. No modo manual o usuário pode, através de um aplicativo para celular ou por um web browser, acionar diretamente o motor da bomba ligando-o ou desligando-o e abrir ou fechar qualquer uma das válvulas. Os sensores de níveis são desprezados, ficando todo o controle com o usuário para ligar e desligar a bomba. O objetivo deste modo é poder operar o sistema caso ocorra problemas com os sensores, deixando de abastecer algum tanque no modo automático.

No modo automático, a aplicação ao detectar o nível baixo em qualquer um dos reservatórios irá abrir a válvula correspondente a ele e acionar o motor da bomba. Neste momento é iniciado o monitoramento da vazão de entrada do reservatório. Caso a vazão permaneça em 0 (zero) $\mathrm{l} / \mathrm{min}$ durante um tempo prédeterminado o sistema irá desligar o motor por segurança, fechar as válvulas e reiniciar o processo de abastecimento. Ao iniciar novamente e permanecendo a vazão em 0 (zero) $\mathrm{I} / \mathrm{min}$ o sistema envia uma mensagem para um e-mail cadastrado informando a condição de que não há fluxo de água e altera a aplicação para modo manual para que o usuário possa testar e identificar o problema.

O abastecimento é realizado de forma individual para evitar sobrecarga ou perda de eficiência do motor.

Durante o processo de abastecimento, o sistema , estando conectado à Internet, envia os status dos equipamentos, o consumo do reservatório em abastecimento e a vazão de água para um cloud cadastrado que armazena todo o histórico de funcionamento do sistema e pode ser acessado por uma página da Internet.

\subsection{A Aplicação}

A aplicação desenvolvida possui interfaces que auxiliam no funcionamento lógico, no controle e no monitoramento da informação. Como ilustrado na Figura 3, o sistema contempla alguns sensores de nível e vazão que enviam sinais ao microcontrolador que é responsável pela tomada de decisões. As saídas do microcontrolador acionam os equipamentos de controle de acordo com os dados de entrada e sua lógica, descrita neste trabalho. o controle e monitoramento são realizados por meio de conexão sem fio padrão $802.11 \mathrm{~B} / \mathrm{G} / \mathrm{N}$.

O sistema completo, nomeado de AquaControle, possui um medidor de vazão do tipo rotâmetro, 4 (quatro) entradas para conexão dos sensores de níveis, 4 (quatro) saídas para ligação das válvulas solenoides, 1 (uma) saída para ligação do motor da bomba de sucção, 1 (uma) fonte de alimentação e um aplicativo para dispositivos com plataforma Android $(B$ como ilustrado na Figura 4 e Figura 5. A seguir está descrito o hardware do AquaControle.

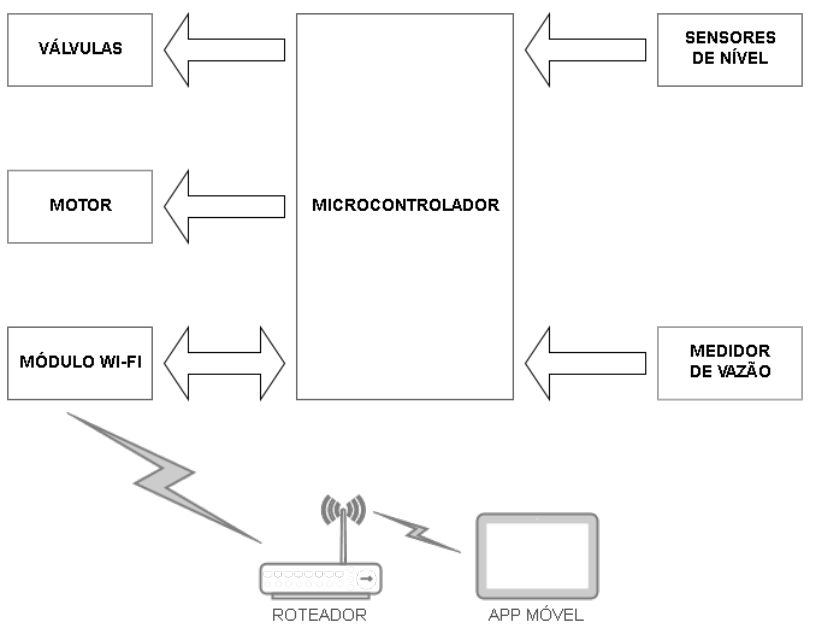

Figura 3: Diagrama de blocos da aplicação.

http://dx.doi.org/10.25286/repa.v2i2.574 


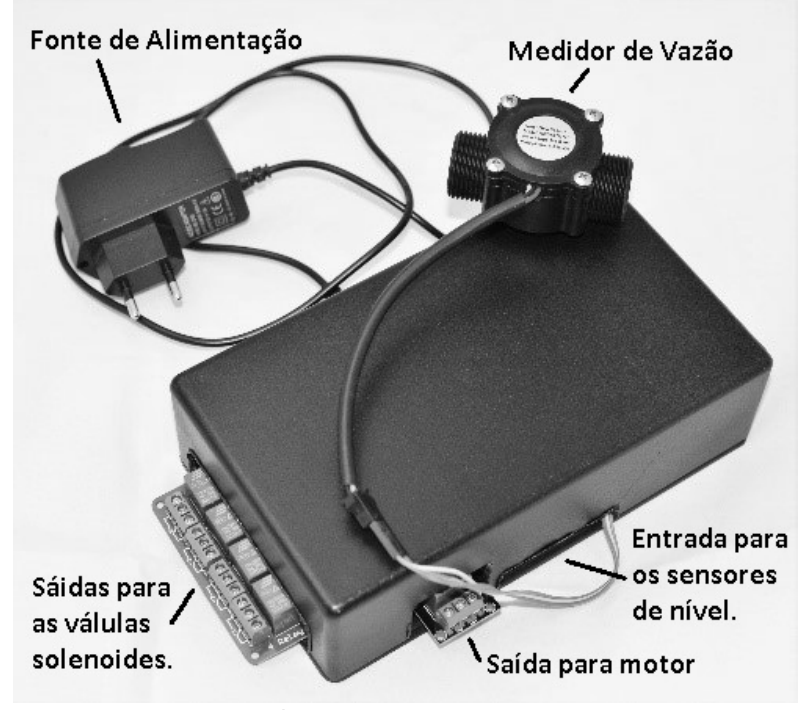

Figura 4: AquaControle.

\section{O Hardware}

Os módulos de desenvolvimento para sistemas embarcados são hoje facilmente encontrados na Internet. Isto ajuda muito para executar um protótipo das aplicações desejadas sem a necessidade de montar os componentes individualmente. O projeto ficou modular facilitando também possíveis manutenções como ilustrado na Figura 5.

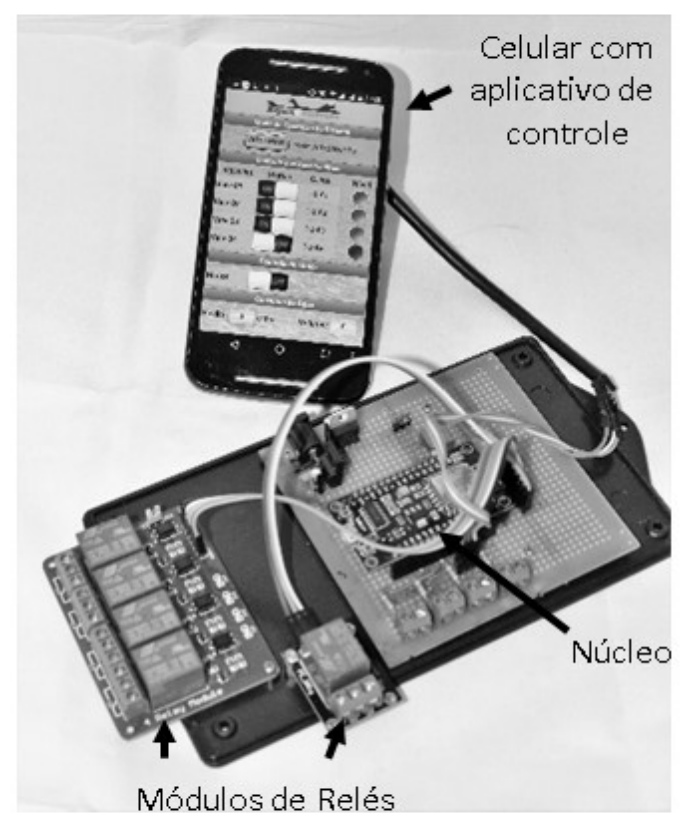

Figura 5: O hardware do AquaControle.

O hardware do AquaControle é composto por um módulo de desenvolvimento NodeMCU, 5 (cinco) módulos de relés e 2 (dois) reguladores de tensão para alimentação dos módulos em $3,3 \mathrm{~V}$ e $5 \mathrm{~V}$. As entradas dos sensores de níveis são ligadas diretamente nas portas digitais do módulo de desenvolvimento.

\subsection{A Aplicação}

O núcleo do hardware tem como base o kit de desenvolvimento NodeMCU que possui o chip ESP8266-12E Este módulo trabalha com um firmware baseado em Lua para o ESP8266 WifiSOC da Espressif [6].

O chip ESP8266-12E é composto pelo microcontrolador Tensilica L106 de 32bits e de baixo consumo de energia, velocidade de clock de $80 \mathrm{MHz}$ e opera com tensões de $3 \mathrm{~V}$ a $3,6 \mathrm{~V}$. Possui módulo WiFi $802.11 \mathrm{~b} / \mathrm{g} / \mathrm{n} / \mathrm{e} / \mathrm{i}$, portas de entrada/saída digitais (GPIOs), comunicação serial (UART), conversor A/D (Analógico/Digital), comunicação serial $\mathrm{I}^{2} \mathrm{C}$ (InterIntegrated Circuit) e $\mathrm{I}^{2} \mathrm{~S}$ (Inter-IC Sound), módulo PWM(Pulse-Width Modulation) e uma controladora para expansão de memória SPIFlash, os quais podem ser apresentados na Erro! Fonte de referência não encontrada. [7].

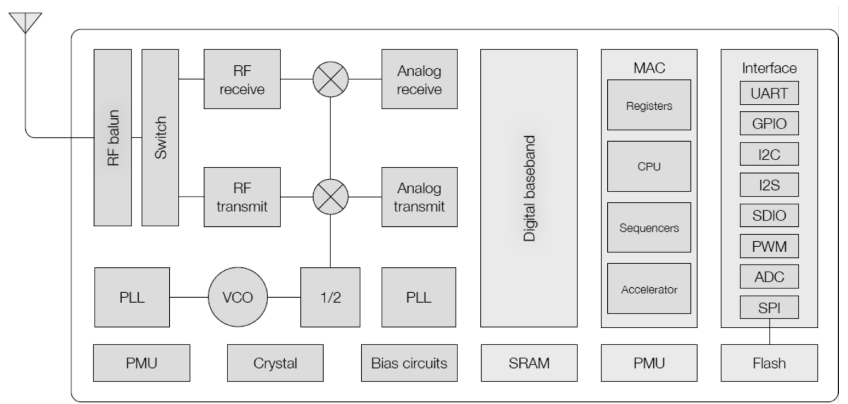

Figura 6: Diagrama de bloco do ESP8266.

Fonte: Ierusalimschy, 2003. [10]

\subsection{Sensores de Entrada}

O sensor é conhecido como um dispositivo que detecta ou mede condições em tempo real, como calor, condição elétrica, movimento ou luz e converteas em uma representação analógica ou digital. A seguir estão descrito os sensores utilizado no projeto. 


\subsubsection{Sensor de Nível}

Os níveis dos reservatórios são monitorados por uma chave de nível acionada por uma bóia, ilustrada na Figura 7, por meio do qual é possível verificar o estado do reservatório, cheio ou vazio.

A informação do estado do reservatório é realizada por um interruptor interno ao dispositivo que envia um nível de tensão para as GPIOs o qual é interpretado pelo código do núcleo, responsável pela tomada as decisões de início ou término do abastecimento.

\subsubsection{Sensor de Fluxo de Água}

Na Figura 8 está ilustrado o sensor de fluxo que é formado por um corpo de plástico, um rotor por onde passa o fluído e o sensor de efeito Hall, que emite um sinal de pulso de acordo com a vazão que passa pelo rotor.

O objetivo deste sensor é medir a vazão de água para que o sistema possa calcular o volume fornecido para cada reservatório, além de servir como um intertravamento de proteção contra falta de fluxo quando o motor estiver ligado, vindo a desligá-lo por segurança do equipamento.

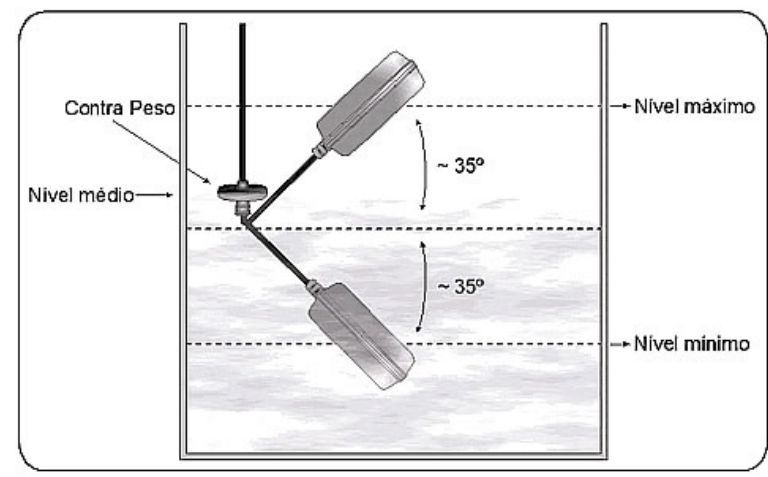

Figura 7: Sensor de nível.

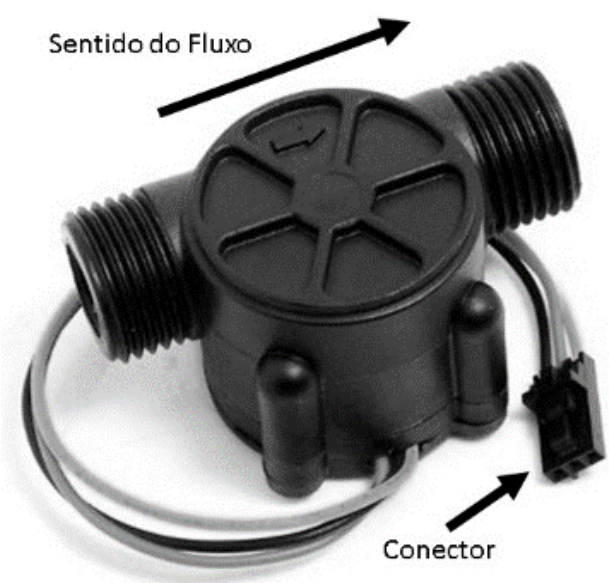

Figura 8: Sensor de fluxo de água.

\subsection{Dispositivos de Saída}

As saídas digitais do NodeMCU possuem níveis de tensão baixos, impossibilitando o acionamento de cargas com alta potência. Por este motivo há necessidade de uma interface entre o módulo de controle e as cargas. Sendo assim, os relés são as melhores alternativas para este acionamento.

\subsubsection{Módulo de Relés}

As válvulas de controle e o motor são acionados pelos relés eletromecânicos, tais como os ilustrados na Figura 9, os quais podem suportar altas correntes. Os relés podem ser atuados com nível lógico alto ou baixo, dependendo do modelo do módulo.

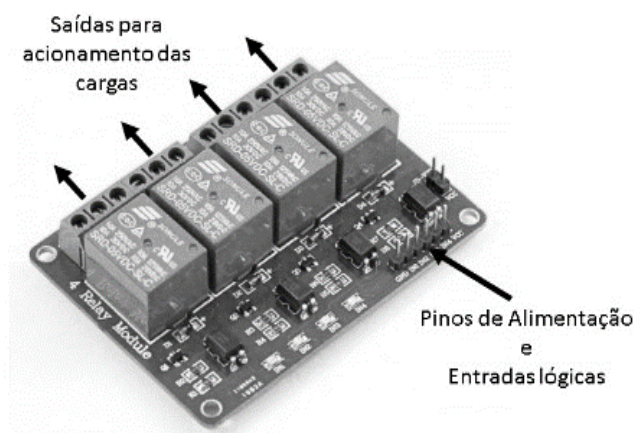

Figura 9: Módulo de 4 relés.

O módulo ilustrado na Erro! Fonte de referência não encontrada. é alimentado por $5 \mathrm{Vcc}$, as entradas http: / / dx.doi.org/10.25286/repa.v2i2.574 
lógicas podem ser acionadas de 3 a 5 Vcc com corrente de operação de 10 a $15 \mathrm{~mA}$.Os relés trabalham com cargas de até 10A/220Vac. [8]

\subsubsection{Válvula Solenoide para Água}

O fluxo de água é direcionado para os reservatórios através das válvulas [9] que são acionadas de acordo com a lógica de controle do sistema caso esteja operando em modo automático ou manual.

A Figura 10 mostra uma válvula solenoide NF (Normalmente fechada) acionada por $220 \mathrm{~V}$.

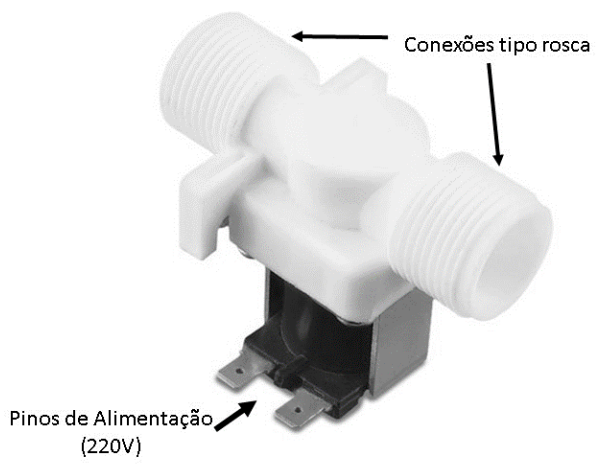

Figura 10: Válvula solenoide.

Quando o relé é acionado, a válvula é alimentada por $220 \mathrm{~V}$ liberando a passagem do fluído em seu interior, no momento em que é desenergizada, a válvula fecha bloqueando a passagem.

\subsubsection{Bomba Centrífuga}

A bomba d'água tem como objetivo bombear a água dos lençóis freáticos para os reservatórios. Este equipamento não pode trabalhar muito tempo com sua descarga fechada, pois poderá aquecer bastante, vindo a danificar seu motor e a bomba. Por este motivo há necessidade de se ter um sistema de proteção para evitar que o equipamento fique ligado com a sua saída de água fechada.

\section{O Software}

Nos sistemas de automação microcontrolado sempre há necessidade de um software para controlar 133 o hardware, neste projeto não seria diferente. É com ele que o núcleo da aplicação analisa os sinais de seus sensores e toma as decisões de ação para alcançar o objetivo do projeto.

O software principal encontra-se na memória flash do chip ESP8266. Ele foi desenvolvido em uma linguagem de script Lua que é bastante utilizada em sistemas embarcados e de fácil adaptação [10].

O NodeMCU possui um firmware que pode ser customizado no site do fabricante [11] de acordo com as funcionalidades do projeto vindo a otimizar o espaço de memória do kit.

Ao ligar o kit, o firmware inicia o boot e executa o arquivo init.lua, neste arquivo deverá estar as primeiras instruções da aplicação, logo em seguida a rotina principal é executada conforme ilustrado na Erro! Fonte de referência não encontrada.

Quando iniciada a rotina principal, o sistema se conecta à rede sem fio já configurada passando para o próximo evento quando conectado, como mostrado na Figura 11. No modo automático, as válvulas e o motor são acionados no momento em que há nível baixo em algum tanque.

Na situação em que outro tanque apresentar nível baixo durante o processo de abastecimento, o sistema altera a abertura das válvulas apenas quando conclui o enchimento do anterior. Estando todos os níveis em condições normais o motor é desligado e a válvula é fechada.

O usuário ao modificar o modo de operação para manual permite que o acionamento das válvulas e do motor seja feitos diretamente pelo aplicativo do celular desconsiderando os níveis dos reservatórios. 


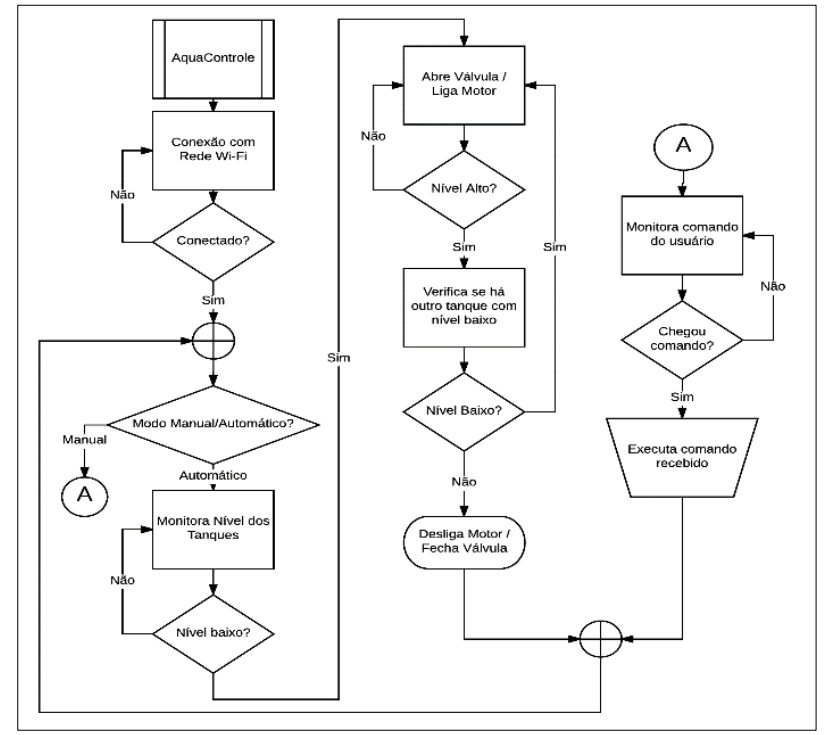

Figura 11: Fluxograma funcional do software do AquaControle.

A qualquer momento o usuário poderá alterar o modo de operação para manual ou automático. Assim, controlando o sistema da melhor forma possível.

\section{O Aplicativo AquaControle}

O aplicativo para os dispositivos móveis foi desenvolvido com o App Inventor [12], uma ferramenta para iniciantes em desenvolvimento de software que utiliza blocos de textos para a construção da lógica. O App Inventor tem como missão transformar os usuário de tecnologia em criadores de tecnologia de uma forma simples e fácil para todos. Esta ferramenta atendeu a necessidade do projeto e deu à aplicação uma aparência bem agradável como observa-se na Figura 12.

A Figura 13 apresenta o diagrama de blocos da aplicação utilizada para enviar um comando ao módulo central através da rede. No primeiro bloco ao acionarmos o botão "on" da Valv 04, o qual pode ser observado na Figura 12(b), a aplicação faz com que ele seja ocultado, torna visível o botão "off" e envia o comando "ON4" para o endereço selecionado na Figura 12(a), este comando faz a Válvula 04 do sistema abrir.
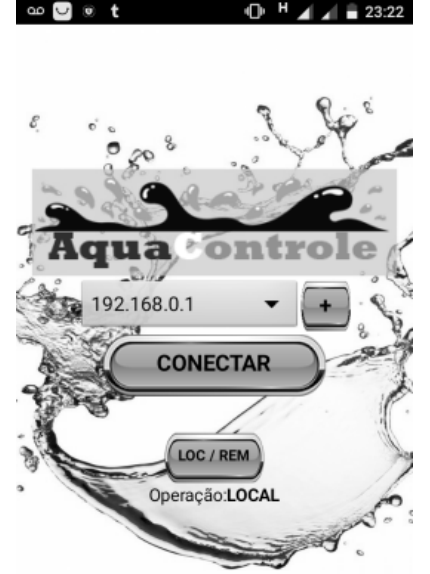

\section{$\triangleleft$} 0 口

Figura 12: (a) Tela de login aplicação.
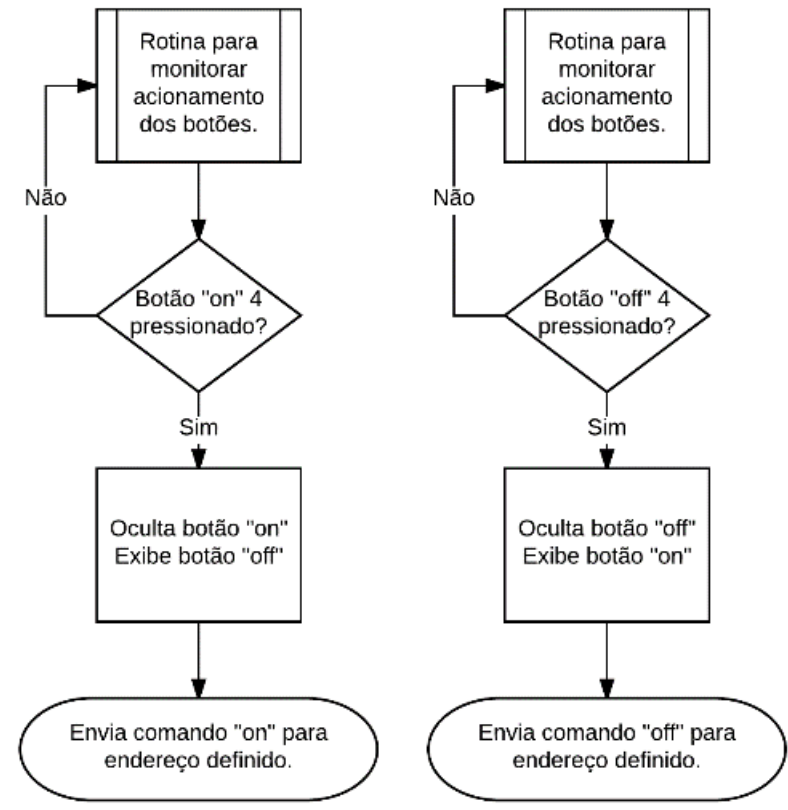

Figura 13: Parte da lógica de bloco da tela de controle.

No segundo bloco a operação é inversa. Ao acionarmos o botão "off" da Valv 04, a aplicação faz com que ele seja ocultado, torna visível o botão "on" e envia o comando "OFF4" onde a Válvula 04 é fechada. (a)

(b) 


\section{Conclusões}

O sistema apresentado foi montado em uma residência que possui o cenário apresentado na Figura 2. A dificuldade encontrada foi a instalação física das chaves de nível devido ao difícil acesso aos reservatórios de água. A distância dos reservatórios não influenciou na leitura do sinal pelas portas digitais do dispositivo. Para o uso do aplicativo AquaControle uma rápida explicação foi suficiente para a utilização o mesmo.

O AquaControle conseguiu alcançar o objetivo proposto neste projeto que era de evitar o desperdício de água com o transbordamento dos reservatórios e disponibilizou uma ferramenta de monitoramento online para os usuários deste sistema.

Com isto, passamos a ter um consumo consciente evitando a retirada indiscriminada das águas subterrâneas e consequentemente preservando os lençóis freáticos.

Outro objetivo alcançado foi a economia de energia com o desligamento do motor no momento em que o nível do reservatório alcança seu valor máximo e também a proteção do motor-bomba caso não haja fluxo de água, seja por falta de escorva ou por fechamento da sua linha de descarga.

Como as saídas de acionamento foram projetadas com relés, este projeto pode ser redirecionada para diversos outras aplicações na automação residencial como por exemplo: acionamentos de lâmpadas, portões eletrônicos, alarmes, cortinas motorizadas, eletrodomésticos entre outros.

\section{Referências}

[1] S. B. Alexandre Luiz , C. F. Waldir Duarte. Atuação da Câmara Técnica de águas subterrâneas - CTAS do Estado de Pernambuco. XVI Congresso Brasileiro de Águas Subterrâneas e XVII Encontro Nacional de Perfuradores de Poços

[2] SANTOS, Maraiza Prescila dos; OLIVEIRA, José Kleber Costa de. AUTOMAÇÃO DE BAIXO CUSTO PARA RESERVATÓRIOS DE ÁGUA, 2014.

[3] Yang, Shuang-Hua, et al. "A case study of Internet of things: A wireless household water consumption monitoring system." Internet of Things (WF-IOT), 2015 IEEE 2nd World Forum on. IEEE, 2015.

135
[4] Oliveira, Isabel RH, Carlos RB Santos, and Marco AL Rodrigues. DESENVOLVIMENTO DE UM APLICATIVO ANDROID PARA MONITORAMENTO MICROCONTROLADO DO NÍVEL DE UM RESERVATÓRIO DE ÁGUA RESIDENCIAL EM TEMPO REAL, 2014.

[5] Abdulrahman, T. A., et al. "Design, Specification and Implementation of a Distributed Home Automation System." Procedia Computer Science 94 (2016): 473-478.

[6] NodeMCU Documenttion. http://nodemcu.readthedocs.io/en/master/en/, Dez 2016.

[7] ESP8266EX Datasheet Version 4.3, http://bbs.espressif.com/, Dez 2016.

[8] Vinitrônica, http://www.vinitronica.com.br/pd-1986b0modulo-rele-4-canais-

5v.html?ct $=$ aba4b\&p=1\&s=1, Abr 2017 .

[9] Vinitrônica, http://www.vinitronica.com.br/pd-19ed1fvalvula-solenoide-3-4-220v.html, Abr 2017.

[10] R. Ierusalimschy, Programming in Lua. Lua.org, December 2003. ISBN 85-903798-1-7.

[11] https://nodemcu-build.com/

[12] MIT App Inventor, http://appinventor.mit.edu/explore/, Dez 2016. 